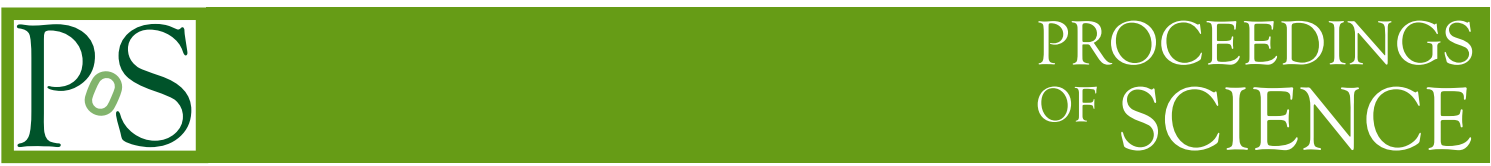

\title{
Charmonium-nucleon interactions from $2+1$ flavor lattice QCD
}

\section{Takuya Sugiura*}

E-mail: sugiuradrcnp.osaka-u.ac.jp

\section{Yoichi Ikeda}

\section{Noriyoshi Ishii}

Research Center for Nuclear Physics (RCNP), Osaka University

\begin{abstract}
The charmonium-nucleon interaction is studied by the time-dependent HAL QCD method. We use a larger lattice volume and the relativistic heavy quark action for charm quark to obtain less systematic errors than those in our previous study. As a result, the sizable $J / \psi N$ hyperfine splitting is observed, indicating that the spin-spin interaction is important to understand this system quantitatively. No $J / \psi N$ or $\eta_{c} N$ bound state is observed below the thresholds as in the previous results.
\end{abstract}

The 36th Annual International Symposium on Lattice Field Theory - LATTICE2018 22-28 July, 2018

Michigan State University, East Lansing, Michigan, USA.

\footnotetext{
* Speaker.
} 


\section{Introduction}

The LHCb collaboration has reported a peak structure in the $J / \psi p$ invariant mass spectrum of the weak decay process $\Lambda_{b} \rightarrow J / \psi p K^{-}$[四]. The two resonances, $P_{c}(4380)$ and $P_{c}(4450)$, are found in the mass spectrum with more than 9 standard deviation significance by the fit with two Breit-Wigner resonances for $P_{c}$ 's and 14 known $\Lambda^{*}$ resonances. The three preferred spin-parity assignments are given as $\left(3 / 2^{-}, 5 / 2^{+}\right),\left(3 / 2^{+}, 5 / 2^{-}\right),\left(5 / 2^{+}, 3 / 2^{-}\right)$for $P_{c}(4380)$ and $P_{c}(4450)$, respectively. Furthermore, the $\mathrm{LHCb}$ recently has analyzed nine times more data than those in the 2015 analysis and found a new resonance $P_{c}(4312)$ and two narrow overlapping peaks $P_{c}(4440)$ and $P_{c}$ (4457) instead of $P_{c}$ (4450) [[]]. Since the $P_{c}$ states are found in the $J / \psi N$ mass spectrum above the threshold, they are naturally expected to have $u u d c \bar{c}$ flavor structure, i.e., the pentaquarks. The discovery of the hidden-charm pentaquarks attracts much attention to explain the origin of these resonances. Since experimental data for interactions between charmed hadrons is very scarce, effective theories will suffer from large ambiguity. In this situation, lattice QCD first-principle calculation of these interactions is of significant importance. Lattice QCD also has an advantage over experimental studies, since we can directly calculate the $J / \psi N$ scattering, without requiring the spectator $K^{-}$.

A possible explanation of $P_{c}$ is that they are loosely bound $\bar{D}^{*} \Sigma_{c}$ and $\bar{D}^{*} \Sigma_{c}^{*}$ states [3], 团]. The interaction between these states is supplied by meson exchange forces. Another picture is proposed in Refs. [ [ $\left[\right.$, 目], where the possibility of having $P_{c}(4450)$ as a deeply bound narrow $\psi(2 S)$-nucleon bound state is discussed. In this scenario, $\psi(2 S)$ is assumed to be a Coulomb bound state, so that the description of the QCD van der Waals force is applicable [ $\square]$. The strength of the QCD van der Waals force is determined by the chromoelectric polarizability of the charmonium, which is approximately proportional to inverse cube of the charmonium radius. Thus the $\psi(2 S) N$ interaction can be very strong and possibly explain as large as $176 \mathrm{MeV}$ binding energy of $P_{c}(4450)$ as a narrow $\psi(2 S) N$ bound state. Although this picture is theoretically interesting, we need to be careful to obtain quantitative understanding what $P_{c}$ 's are like. In Ref. [8], results of our previous calculation [Q] have been used for a rough, but quantitative evaluation of the $J / \psi$ chromoelectric polarizability. In this present paper, we study the $J / \psi N$ and $\eta_{c} N$ scattering in lattice QCD with better statistics and systematics than those in Ref. [Q].

As before, we employ the method developed by the HAL QCD collaboration [प], प]]. The HAL QCD method can be straightforwardly extended to coupled-channel systems [ए2]; this is an important advantage over Lüscher's finite volume method [[13]. We have improved the calculations by Kawanai and Sasaki [ [4]] by using the time-dependent HAL QCD method and found that the charmonium-nucleon interaction has significantly stronger attraction than their results. We have also found significant hyperfine splitting of the $J / \psi N$ interaction between the $J=1 / 2$ and $J=$ $3 / 2$ states. This paper is organized as follows. In Sec. \, we briefly introduce the HAL QCD (both original and time-dependent), mentioning possible origins of systematic errors and necessary

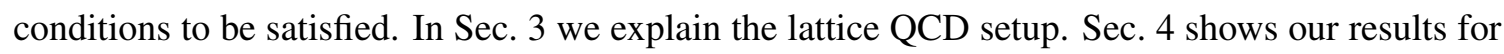
the $J / \psi N$ and $\eta_{c} N$ interactions. We discuss importance of using the time-dependent method by taking a close look at the terms appearing by temporal derivatives. We summarize our conclusions in Sec. [.]. 


\section{Method}

The charmonium-nucleon four point function is defined as

$$
\begin{aligned}
R\left(\boldsymbol{x}-\boldsymbol{y}, t-t_{0}\right) & =\left\langle 0\left|T \phi(\boldsymbol{x}, t) N(\boldsymbol{y}, t) \overline{\mathscr{J}}\left(t_{0}\right)\right| 0\right\rangle \times e^{\left(m_{N}+m_{\phi}\right) t} \\
& =\sum_{n} A_{n} \psi_{n}(\boldsymbol{r}) e^{-\Delta W_{n} t}
\end{aligned}
$$

where $\phi(\boldsymbol{y}, t)$ and $N(\boldsymbol{x}, t)$ are local interpolating operators for charmonium (either $J / \psi$ or $\eta_{c}$ ) and nucleon, respectively, and $\mathscr{J}\left(t_{0}\right)$ is the corresponding wall-source operator. The masses of $\phi$ and $N$ are denoted as $m_{\phi}$ and $m_{N}$. The second line can be derived by inserting the complete set of the QCD eigenstates $\mathbf{1}=\sum_{n}|n\rangle\langle n|$ and by defining $\psi_{n}(\boldsymbol{r})=\langle 0|\phi(\boldsymbol{r}+\boldsymbol{x}, 0) N(\boldsymbol{x}, 0)| n\rangle, A_{n}=\langle n|\overline{\mathscr{J}}(0)| 0\rangle$, the $n$-th eigenvalue $W_{n}=\sqrt{k_{n}^{2}+m_{\phi}^{2}}+\sqrt{k_{n}^{2}+m_{N}^{2}}$ and $\Delta W_{n}=W_{n}-W_{0} . \psi_{n}(\boldsymbol{r})$ is called the equaltime Nambu-Bethe-Salpeter (NBS) wave function, as it satisfies the Helmholtz equation in the long-distance limit $|\boldsymbol{r}| \rightarrow \infty$. The energy-independent and non-local potential $U\left(\boldsymbol{r}, \boldsymbol{r}^{\prime}\right)$ is defined by [ए]]

$$
\left(\frac{\nabla^{2}}{2 \mu}+E_{n}\right) \psi_{n}(\boldsymbol{r})=\int d^{3} \boldsymbol{r}^{\prime} U\left(\boldsymbol{r}, \boldsymbol{r}^{\prime}\right) \psi_{n}\left(\boldsymbol{r}^{\prime}\right),
$$

where $\mu=1 /\left(1 / m_{\phi}+1 / m_{N}\right)$ is the reduced mass and $E_{n}=k_{n}^{2} /(2 \mu)$. In the original HAL QCD method, one solves Eq. (2.3]) for the potential $U$; for that one has to extract the NBS wave function for the ground state by assuming the ground-state saturation, i.e., $R(\boldsymbol{r}, t) \simeq A_{0} \psi_{0}(\boldsymbol{r}) e^{-\Delta W_{0} t}$ at large $t$. In actual situations, ground-state saturation is hard to achieve because the signal-to-noise ratio becomes exponentially bad for large $t$. An alternative method has been developed in Ref. [U]]. The same potential $U\left(\boldsymbol{r}, \boldsymbol{r}^{\prime}\right)$ satisfies the following time-dependent Schrödinger-like equation upto $\mathscr{O}\left(k_{n}^{6}\right)$ :

$$
\left(\frac{\nabla^{2}}{2 \mu}-\frac{\partial}{\partial t}+\frac{1+3 \delta^{2}}{8 \mu} \frac{\partial^{2}}{\partial t^{2}}\right) R(\boldsymbol{r}, t)=\int d^{3} \boldsymbol{r}^{\prime} U\left(\boldsymbol{r}, \boldsymbol{r}^{\prime}\right) R\left(\boldsymbol{r}^{\prime}, t\right),
$$

where $\delta=\left(m_{\phi}-m_{N}\right)\left(m_{\phi}+m_{N}\right)$. Eq. (2.4) is satisfied upto inelastic state contributions; in other words, one requires that the four-point function is dominated by the states with energy below the threshold energy of the next channel $W_{t h}$ (elastic-state saturation). In our lattice setup with heavy pion mass, that is $W_{t h}=m_{D}+m_{\Sigma_{c}}$. The elastic-state saturation is much easier to achieve than the ground-state saturation, and one can reliably extract the potentials by Eq. (2.4). By taking the lowest-order term of the derivative expansion, i.e. $U\left(\boldsymbol{r}, \boldsymbol{r}^{\prime}\right) \simeq V_{\mathrm{eff}}(r) \delta^{3}\left(\boldsymbol{r}-\boldsymbol{r}^{\prime}\right)^{1}$, one can calculate the effective central potential as

$$
V_{\mathrm{eff}}(r)=\frac{1}{2 \mu} \frac{\nabla^{2} R(\boldsymbol{r}, t)}{R(\boldsymbol{r}, t)}-\frac{\partial_{t} R(\boldsymbol{r}, t)}{R(\boldsymbol{r}, t)}+\frac{1+3 \delta^{2}}{8 \mu} \frac{\partial_{t}^{2} R(\boldsymbol{r}, t)}{R(\boldsymbol{r}, t)} .
$$

\footnotetext{
${ }^{1}$ Note that the $J / \psi N$ potential consists of the central, the spin-spin, and two tensor forces at the lowest order of the derivative expansion. We can determine each of these four forces by using linearly-independent correlation functions for four different states. Then we find that the S-D wave orbital angular momentum mixing due to the tensor forces is much smaller than the central and the spin-spin forces, so that we can tentatively work without considering S-D mixing explicitly. The detailed results of the spin-dependent forces are presented elsewhere.
} 
Although the right-hand side seems to depend on $t$, the left-hand side does not as far as the elasticstate saturation is achieved and the derivative expansion converges at this order. Therefore, by seeing the $t$-independence of $V_{\text {eff }}(r)$ we can check the necessary condition for our assumptions: the elastic-state saturation and the convergence of the derivative expansion. Moreover, when the ground-state saturation is achieved, the time-derivative terms do not depend on the spatial distance $r$; the $\left(\partial_{t} R\right) / R$ term roughly corresponds to $E_{n}$ and the $\left(\partial_{t}^{2} R\right) / R$ term corresponds to non-relativistic correction of $\mathscr{O}\left(k_{n}^{4}\right)$. Although we do not need the ground-state saturation anymore, this property will help us understand our results in comparison to previous calculations based on the original HAL QCD method.

In this paper, we neglect the coupling of $J / \psi N$ and $\eta_{c} N$, since such a charmonium spinflipping transition is suppressed due to the large charm quark mass. We also neglect the OZIsuppressed $c \bar{c}$ annihilation diagrams to calculate the four-point function in Eq. ([2.2).

\section{Simulation Setup}

We employ the $2+1$ flavor QCD gauge configurations on a $32^{3} \times 64$ lattice, which is generated by the PACS-CS collaboration [[5] with the renormalization group improved gauge action at $\beta=1.9$ and the non-perturbatively $\mathscr{O}(a)$ improved Wilson quark action at $c_{S W}=1.715$. The corresponding lattice spacing is $a=0.0907(13) \mathrm{fm}$ and the spatial volume is $L=(2.90 \mathrm{fm})^{3}$. We use the hopping parameters $\kappa_{\mathrm{ud}}=0.13700$ and $\kappa_{\mathrm{s}}=0.13640$. For the charm quark we employ the Tsukuba-type relativistic heavy quark (RHQ) action to remove the leading and next-to-leading order cutoff errors [ए6]. We use the RHQ parameters determined in Ref. [ए7] such that physical hadron properties are reproduced at physical quark masses. The periodic boundary condition is imposed in the spatial directions, while the Dirichlet boundary condition is imposed in the temporal direction at $\left(t-t_{0}\right) / a=32$ to prevent inverse propagation. We use 32 different source positions $t_{0}$ and take their average.

\section{Results and Discussion}

Shown in Fig. $\square$ are the effective masses $m_{\mathrm{eff}}(t)=(1 / a) \ln \left(C_{2}(t) / C_{2}(t+a)\right)$ for $J / \psi, \eta_{c}$, and $N$ calculated from the corresponding hadron two-point functions $C_{2}(t)$. The masses are evaluated by fitting the two-point functions by a single exponential in the plateau region of the effective mass plots. We find that $m_{J / \psi}=3139(12) \mathrm{MeV}\left(\left(t-t_{0}\right) / a=15-18\right), m_{\eta_{c}}=3022(9) \mathrm{MeV}\left(\left(t-t_{0}\right) / a=\right.$ $15-18)$, and $m_{N}=1585(16) \mathrm{MeV}\left(\left(t-t_{0}\right) / a=14-19\right)$, where the numbers in the first parentheses are statistical errors, and the second parentheses indicate the fit ranges. Similar analysis for pion shows $m_{\pi}=700(2) \mathrm{MeV}\left(\left(t-t_{0}\right) / a=11-18\right)$.

In Fig. 四, we show the S-wave effective central potentials for $J / \psi N(J=1 / 2), J / \psi N(J=$ $3 / 2)$, and $\eta_{c} N$ evaluated at $\left(t-t_{0}\right) / a=15$. We see that all of them are attractive overall and the $J / \psi N$ interactions (both $J=1 / 2$ and $J=3 / 2$ ) are stronger than the $\eta_{c} N$ interaction, as already reported previously [Q [4] [4]. We also observe the hyperfine splitting of $J / \psi N$ interactions; the $J=1 / 2$ state has stronger attraction than the $J=3 / 2$ state. In Ref. [Q], the hyperfine splitting is much smaller than the difference between $J / \psi N$ and $\eta_{c} N$ potentials, and than the absolute value of the $J / \psi N$ potential. In this study we use a larger lattice with volume $L a=2.90 \mathrm{fm}$ than the one 

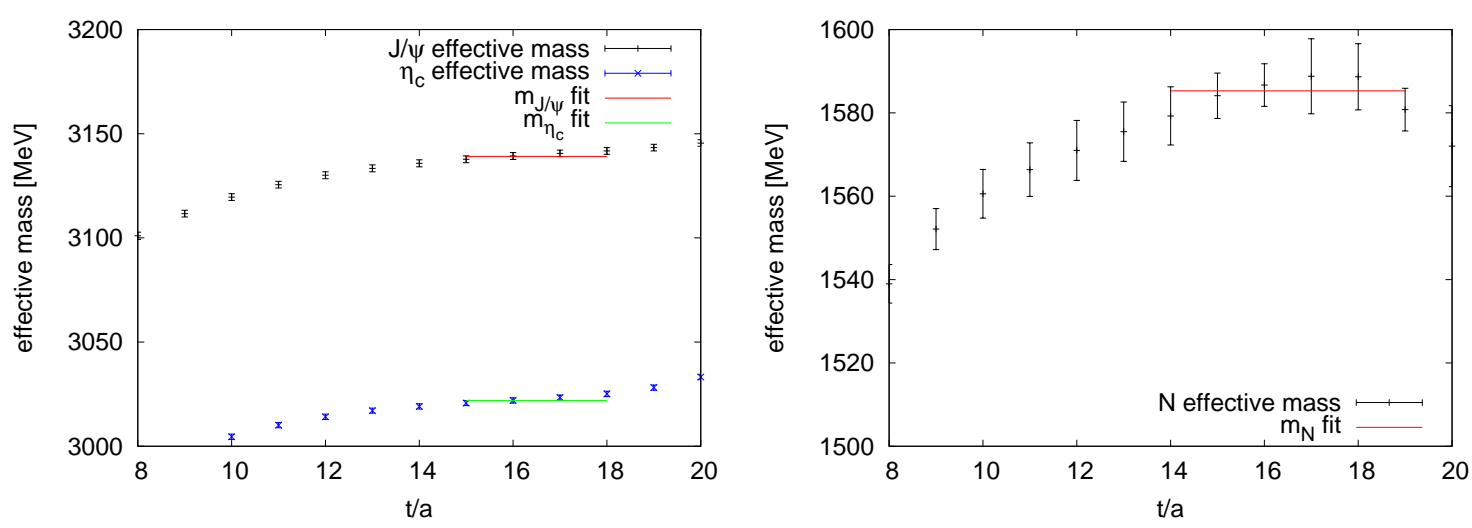

Figure 1: Effective masses for (left) $J / \psi$ and $\eta_{c}$ and (right) nucleon.

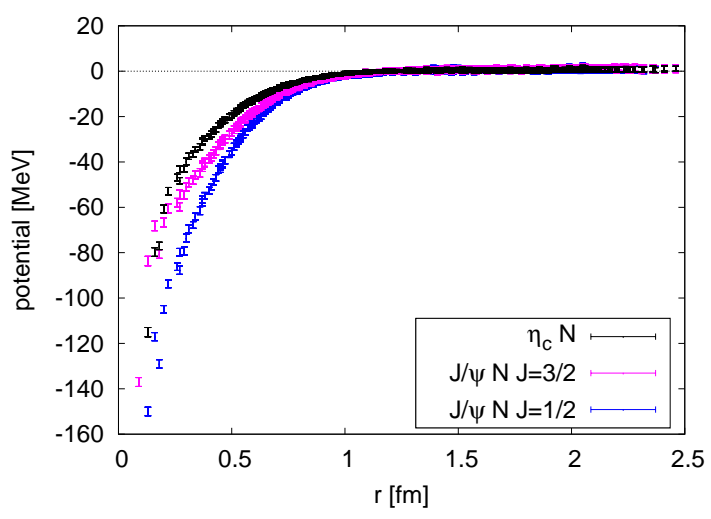

Figure 2: The $\mathrm{S}$-wave effective central potentials for (blue) $J / \psi N(J=1 / 2)$, (magenta) $J / \psi N(J=3 / 2)$, and (black) $\eta_{c} N$ evaluated at $\left(t-t_{0}\right) / a=15$.

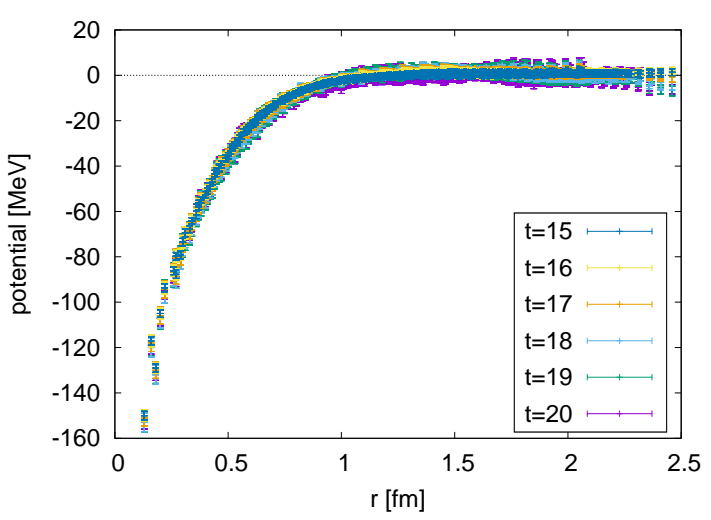

Figure 3: The Euclidean time dependence of the $J / \psi N(J=1 / 2)$ effective central potential.

in Ref. [Q] with $L a=1.93 \mathrm{fm}$. Also, in this study we employ the RHQ action for charm quark to reduce discretization errors, while in Ref. [Q] we do not. Since the $J / \psi N$ interaction is short-ranged such that $V_{\text {eff }}(r) \simeq 0$ for $r>1 \mathrm{fm}$, the finite volume effect should not be important. Thus it seems that we can now see the $J / \psi N$ hyperfine splitting clearly thanks to the use of the RHQ action. The origin of the hyperfine splitting is the spin-spin force, which arises due to interference of the chromoelectric dipole and the chromomagnetic quadrupole in the QCD van der Waals framework.

In order for the potential to be correct, we have to confirm that the elastic-state saturation for the R-correlator in Eq. (․2.) and that the derivative expansion converges at this order. These assumptions can be checked by seeing the $t$-independence of the resulting potential. To do this ${ }^{2}$, in Fig. [1], we show the $J / \psi N(J=1 / 2)$ effective central potentials evaluated at different $t-t_{0}$. We see no significant $t$-dependence in the potential from $\left(t-t_{0}\right) / a=15$ to 20 , so that our necessary condi-

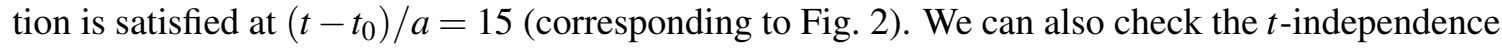
of the $J / \psi N(J=3 / 2)$ and $\eta_{c} N$ potentials.

\footnotetext{
${ }^{2}$ Rigorously speaking, the lowest-order $J / \psi N$ potential consists of the central, spin-spin, and two tensor forces, so that the $t$-independence of each of these forces should be checked. Here we show the $t$-independence of the effective central potential instead, but we have also confirmed the $t$-independences of the four forces explicitly.
} 
The effective central potentials are short-ranged, so that they should receive little finite volume effect. The extrapolation to the infinite volume can thus be easily done, by fitting the potentials with a function that goes to zero quickly as $r$ increases. We employ a fit by two Gaussian functions, $V_{\text {eff }}(r)=\sum_{n=1,2} v_{n} \exp \left(-a_{n} r^{2}\right)$ in all cases. Then they are used to solve the effective radial Schrödinger equation for the scattering phase shift. In Fig. 团, we show the scattering phase shifts thus calculated as a function of the center-of-mass energy $E=k^{2} /(2 \mu)$. No $J / \psi N$ or $\eta_{c} N$ bound state is observed below the thresholds. This observation is consistent with the previous HAL QCD calculations [Q, [4] ] and another study in the Lüscher's formalism [18]]. Interestingly, the NPL collaboration has reported a $\eta_{c} N$ bound state with binding energy $19.7 \mathrm{MeV}$ [ए耳] at the flavor SU(3) point with $m_{\pi}=807 \mathrm{MeV}$, contradicting our conclusion. The low-energy S-matrix elements are well parameterized by the scattering length $a$ and the effective range $r$, defined through the effective range expansion $k \cot \delta_{0}(k)=1 / a+r k^{2}+\mathscr{O}\left(k^{4}\right)$. Neglecting the $\mathscr{O}\left(k^{4}\right)$ contributions, we get $a$ and $r$ for the charmonium-nucleon scattering as in Table. $\mathbb{~}$.

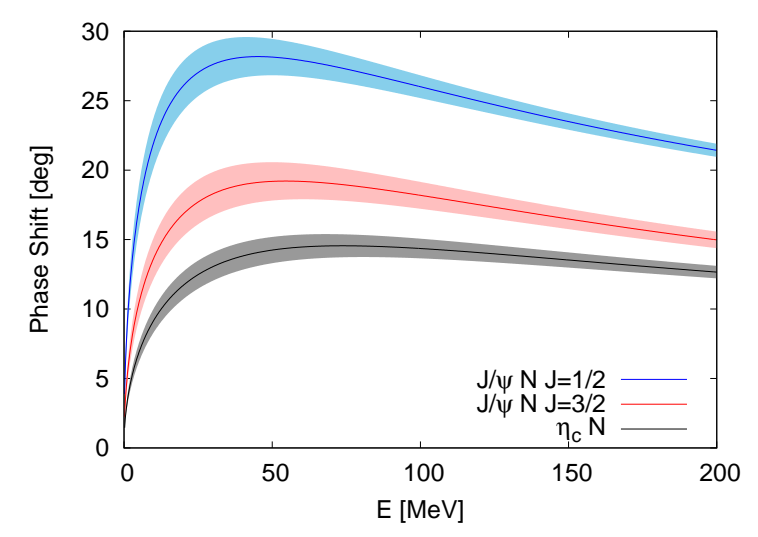

Table 1: The scattering length and the effective range from the effective central potentials.

\begin{tabular}{|c|c|c|}
\hline channel & $a[\mathrm{fm}]$ & $r[\mathrm{fm}]$ \\
\hline$J / \psi N, J=1 / 2$ & $0.656 \pm 0.071$ & $1.105 \pm 0.016$ \\
\hline$J / \psi N, J=3 / 2$ & $0.380 \pm 0.048$ & $1.476 \pm 0.039$ \\
\hline$\eta_{c} N$ & $0.246 \pm 0.026$ & $1.703 \pm 0.045$ \\
\hline
\end{tabular}

Figure 4: S-wave scattering phase shifts calculated from the potentials in Fig. $\square$ as a function of the centerof-mass energy.

\section{Conclusions}

We have studied the $S$-wave charmonium-nucleon scattering by using the time-dependent HAL QCD method. The $J / \psi N(J=1 / 2$ and $J=3 / 2)$ and the $\eta_{c} N$ potentials are all attractive overall, in qualitative agreement with previous HAL QCD calculations. In this study, we have found that the $J=1 / 2 J / \psi N$ state obtains significantly stronger attraction than the $J=3 / 2$ state. This will be useful to discuss the applicability of the QCD van der Waals framework to the charmoniumnucleon systems, since the hyperfine splitting is an $\mathscr{O}\left(1 / m_{c}\right)$ effect and is usually neglected in model calculations. Quantitative evaluation of the QCD van der Waals framework is important to see $P_{c}$ as a $\psi(2 S) N$ bound state. Calculations with lighter pion masses are also necessary for this purpose. The coupled-channel analysis for the $\bar{D}^{(*)} \Sigma_{c}^{(*)}$ channels is important for a theoretical search of $P_{c}$ based on the hadronic molecular picture. This will be left as our next subject. 


\section{Acknowledgements}

This work was supported by JSPS Grant-in-Aid for Scientific Research (S), No. 18H0523, Japan Society for the Promotion of Science KAKENHI Grands No. JP25400244 and by Ministry of Education, Culture, Sports, Science and Technology as "Priority Issue on Post-K computer" (Elucidation of the Fundamental Laws and Evolution of the Universe) and Joint Institute for Computational Fundamental Science. We thank PACS-CS Collaboration [115] and ILDG/JLDG [20] for providing the gauge configurations. The numerical calculations have been performed on OakforestPACS at the University of Tokyo and OCTOPUS in Osaka University. The lattice QCD code is partly based on Bridge++ [2]].

\section{References}

[1] R. Aaij et al. [LHCb Collaboration], Phys. Rev. Lett. 115, 072001 (2015).

[2] https://home.cern/news/news/physics/ lhcb-experiment-discovers-new-pentaquark

[3] L. Roca and E. Oset, Eur. Phys. J. C 76, no. 11, 591 (2016).

[4] Y. Shimizu and M. Harada, Phys. Rev. D 96, no. 9, 094012 (2017).

[5] M. I. Eides, V. Y. Petrov and M. V. Polyakov, Phys. Rev. D 93, no. 5, 054039 (2016).

[6] M. I. Eides, V. Y. Petrov and M. V. Polyakov, Eur. Phys. J. C 78, no. 1, 36 (2018).

[7] M. E. Peskin, Nucl. Phys. B 156, 365 (1979). G. Bhanot and M. E. Peskin, Nucl. Phys. B 156, 391 (1979).

[8] M. V. Polyakov and P. Schweitzer, Phys. Rev. D 98, no. 3, 034030 (2018).

[9] T. Sugiura, Y. Ikeda and N. Ishii, EPJ Web Conf. 175, 05011 (2018).

[10] N. Ishii, S. Aoki and T. Hatsuda, Phys. Rev. Lett. 99, 022001 (2007).

[11] N. Ishii et al. [HAL QCD Collaboration], Phys. Lett. B 712, 437 (2012).

[12] S. Aoki et al. [HAL QCD Collaboration], PTEP 2012, 01 A105 (2012).

[13] M. Luscher, Nucl. Phys. B 354, 531 (1991).

[14] T. Kawanai and S. Sasaki, Phys. Rev. D 82, 091501 (2010).

[15] S. Aoki, et al. [PACS-CS Collaboration], Phys. Rev. D 79, 034503 (2009). S. Aoki, et al. [PACS-CS Collaboration], Phys. Rev. D 81, 074503 (2010).

[16] S. Aoki, Y. Kuramashi and S. i. Tominaga, Prog. Theor. Phys. 109, 383 (2003)

[17] Y. Namekawa et al. [PACS-CS Collaboration], Phys. Rev. D 84, 074505 (2011).

[18] U. Skerbis and S. Prelovsek, arXiv:1811.02285 [hep-lat].

[19] S. R. Beane, E. Chang, S. D. Cohen, W. Detmold, H.-W. Lin, K. Orginos, A. Parreño and M. J. Savage, Phys. Rev. D 91, no. 11, 114503 (2015).

[20] T. Amagasa et al., J. Phys. Conf. Ser. 664, 042058 (2015). http://www.lqcd.org/ildg, http://www.jldg.org

[21] http://bridge.kek.jp/Lattice-code/ 